\title{
A massa máxima das estrelas de nêutrons: uma abordagem didática
}

The maximum mass of a neutron star: a didactic approach

\author{
J.E. Horvath*1] \\ ${ }^{1}$ Universidade de São Paulo, Instituto de Astronomia, Geofísica e Ciências Atmosféricas,Departamento de Astronomia, Cep \\ 05508-090, São Paulo, SP, Brasil.
}

Recebido em 08 de junho de 2020. Revisado em 25 de junho de 2020. Aceito em 28 de junho de 2020.

\begin{abstract}
Embora seja um tópico muito interessante a ser discutido nos cursos introdutórios de Astronomia, as estrelas de nêutrons são muitas vezes apresentadas como uma espécie de "anomalia" astronômica, e sua física e estrutura quase totalmente ignoradas. Encontram-se amiúde referências equivocadas à "massa de Chandrasekhar"ligadas a elas que levam a uma grande confusão. Utilizamos em este trabalho a forma mais simples do equilíbrio hidrostático e uma discussão elementar das forças nucleares para mostrar 1) que as maiores massas das estrelas de nêutrons, onde a matéria está em um regime extremo, nada têm a ver com a massa de Chandrasekhar; 2) que uma análise elementar do equilíbrio estelar permite inferir as soluções tal como são determinadas pelas forças nucleares repulsivas e 3) que essas soluções implicam massas máximas compatíveis com as medidas recentes de estrelas de nêutrons $>2 M_{\odot}$ (isto último claramente impossível para a "massa de Chandrasekhar").
\end{abstract}

Palavras-chave: Estrelas de nêutron, Equilíbrio hidrostático, Forças nucleares.

In spite of being a very interesting topic to be discussed in the introductory courses of Astronomy, neutron stars are often presented as a kind of astronomical "anomaly", and their physics and structure ignored. It is not uncommon to find misleading references to the "Chandrasekhar's mass" linked to them, leading to considerable confusion. We use in this work the simplest form of hydrostatic equilibrium and an elementary discussion of nuclear forces to show 1) that the masses of neutron stars, where matter is found in an extreme regime, are unrelated to Chandrasekhar's mass; 2) that an elementary analysis of stellar equilibrium allows and inference of the solutions as determined by repulsive nuclear forces and 3) that the values of the maximum masses so obtained are in agreement with the most recent measurements of actual neutron stars having $>2 M_{\odot}$ (the latter clearly impossible for the Chandrasekhar's mass).

Keywords: Neutron stars, Hydrostatic equilibrium, Nuclear forces.

\section{Introdução: As estrelas de nêutrons}

Nas primeiras décadas do século 20 o estudo das estrelas foi alavancado pela consolidação da Mecânica, a Termodinâmica o Eletromagnetismo (que já tinham avançado muito no século anterior), e pela emergência da Mecânica Quântica e a Relatividade. O principal exemplo disto é a história das anãs brancas, um novo tipo de estrutura estelar que somente tem sentido quando acionada a Física Quântica [1] na descrição do estado da matéria, indo além do regime de gás clássico utilizado para a Sequência Principal e estágios posteriores [2]. A grande sistematização inicial da teoria das anãs brancas teve como protagonista fundamental a S. Chandrasekhar e está sintetizada no seu texto clássico [3]. Entre outros resultados fundamentais, Chandrasekhar estabeleceu que, no regime quântico onde a pressão é principalmente devida aos elétrons degenerados, existe uma massa máxima, numericamente $\sim 1.4 M_{\odot}$ que pode ser suportada pela estrutura. Esta massa, fisicamente realizada no fim da sequência de anãs brancas frias, leva seu nome e é um conceito importante em toda a Evolução Estelar.

A importância da "massa de Chandrasekhar" na Evolução Estelar, com presença prévia à formação das anãs brancas nas estrelas de tipo solar, é evidente quando notamos que os caroços de estrelas de baixa massa podem entrar em degenerescência (onde a Física Quântica e não a agitação térmica clássica determina as propriedades) e produzir fenômenos evolutivos importantes, tais como o flash de hélio, no qual o limiar de fusão de hélio encontra a matéria em condições degeneradas no topo do Ramo das Gigantes [2]. Desta forma, quando as reações começam, a liberação de energia não afeta a estrutura (já que a matéria degenerada não reage ao aumento de temperatura) até que a potência liberada atinge valores estimados em $10^{10} L_{\odot}$ ou mais em tempos muito curtos, mas possivelmente fica restrita ao interior e esmaecida antes de chegar à superfície. Até agora não houve como

*Endereço de correspondência: foton@iag.usp.br. 
conferir esta predição do flash, amplamente aceita como inferência teórica.

O outro processo no qual a degenerescência eletrônica tem um papel importante é no fim da vida das estrelas de grande massa. Na literatura é descrito como cada ciclo nuclear é aceso como decorrência da contração do caroço com o elemento inerte ( $\mathrm{He}, \mathrm{C}$, etc. sucessivamente), até que as reações de fotodesintegração conseguem extrair energia do rearranjo do $S i$ (na verdade, um conjunto de clusters com os números de núcleos de silício [2]) em elementos do chamado pico do ferro em torno de $A=56$. Esse caroço de "Fe"cresce e está suportado pelos elétrons degenerados. Quando atingidas as condições de chegar à captura eletrônica e fotodesintegração, a pressão desaba e o caroço entra em colapso. É muito possível que seja esta sequência de eventos a que leve à interpretação generalizada de que o caroço, depois da formação da onda de choque e explosão, ao se transformar na estrela de nêutrons "mantenha impressa"a massa que tinha ao começar o colapso. 1

Mas isto não é realmente correto: antes de mais nada o caroço não é uma "anã branca", a temperatura é alta e existem várias correções importantes que devem ser feitas. Assim, os caroços podem crescer até $\sim 2 M_{\odot}$ como observado nas simulações numéricas, o qual leva à formação de estrelas de nêutrons de até $\sim 1.8 M_{\odot}$ de forma "direta", após a radiação de $\sim 10 \%$ da massa para ficar ligado e frio, e sem considerar os casos onde a formação de um buraco negro é o desfecho do colapso $[4]$.

Em suma, não é verdade que as massas das estrelas de nêutrons seja atrelada ao "limite de Chandrasekhar"dos caroços de $\mathrm{Fe}$ progenitores. Estes últimos têm massas típicas entre $1.2 M_{\odot}$ e $1.8 M_{\odot}$ aproximadamente, uma faixa bem mais ampla que o $1.4 M_{\odot}$ amplamente difundido.

Existe porém uma razão ainda mais forte para desvincular a massa de Chandrasekhar das estrelas de nêutrons. A especulação original de L. Landau a respeito das estrelas de nêutrons [5] (que na verdade, precedeu a descoberta do próprio nêutron, vide Ref.[6] para uma análise completa e documentada) contém uma estimativa da massa máxima que poderiam ter esses objetos, então hipotéticos, calculada com a equação de estado que supõe os nêutrons livres, sem nenhuma interação. O resultado numérico é $M_{\max }^{\operatorname{Landau}}=0.7 M_{\odot}$, um valor que so foi testado décadas depois. Foi precisamente depois das descoberta dos pulsares [7], identificados com as estrelas de nêutrons de Landau, e mais precisamente, quando descobertos os sistemas que contém pulsares em binárias (Ref.[8] e literatura citada nela) que a massa destes foi medida, dando origem a crença de que o valor $1.4 M_{\odot}$ era denominado "canônico"ao serem observados alguns sistemas duplos com estrelas de nêutrons nesse patamar. Mas é impor-

\footnotetext{
${ }^{1}$ Ao longo deste trabalho as massas são medidas/referenciadas à massa do Sol, $M_{\odot}=1,99 \times 10^{33} \mathrm{~g}$, os raios ao raio solar , $R_{\odot}=6,96 \times 10^{10} \mathrm{~cm}$ e a luminosidade (potência) ao valor solar $L_{\odot}=3.83 \times 10^{33} \mathrm{ergs}^{-1}$.
}

tante observar que, mesmo se isto estivesse correto, as medidas disponíveis há $~ 50$ anos já eram incompatíveis com uma massa de $0.7 \mathrm{M} \odot$. Assim, os nêutrons livres, equivalente de alta densidade aos elétrons livres para sustentar a estrutura, não fornecem explicação alguma para as observações (Figura 1).

É importante apontar que existem hoje fortes evidências da existência de massas bem maiores que $\sim 1.4 M_{\odot}$ , obtidas por métodos variados, e que mostram que a distribuição de massas é mais complexa [10] que um simples máximo centrado naquela massa. A lista de estrelas de nêutrons com massas altas, cada vez mais próximas do limite de Rhoades-Ruffini, cresceu e isto leva a pensar que é imprescindível que a equação de estado seja capaz de suportar estes valores para a $M_{\max }$ para ter sentido. A Tabela 1 mostra alguns destes casos confirmados, ainda com erros consideráveis, mas que requer nos interiores estelares fontes de pressão suficiente, bem além da fornecida pelos nêutrons livres de Landau. Este tema será objetivo da próxima seção.

\section{O regime supranuclear da matéria e as forças nucleares}

A Física Nuclear desenvolveu até a década de 1970 uma ampla gama de modelos das forças nucleares entre núcleons. Esta tarefa tinha começado com Yukawa [15] em 1935 com a proposta de um mediador das interações cuja massa determinava o alcance. O méson pi de Yukawa ficou associado às interações atrativas que ligam o núcleo, mas a evidência posterior mostrou uma forte repulsão em experimentos de espalhamento quando distâncias menores eram atingidas. Era natural postular que esta

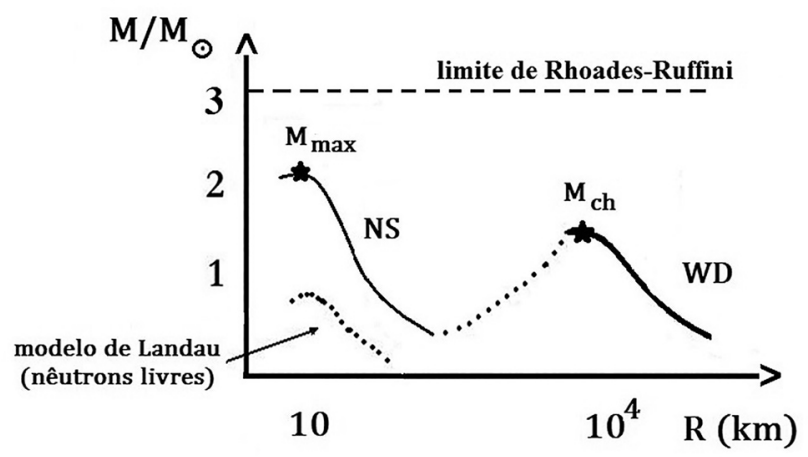

Figura 1: As sequências das anãs brancas (WD, direita) e das estrelas de nêutrons (NS, esquerda) no plano M-R. As estrelas pretas marcam o fim da sequência teórica para cada caso, a massa de Chandrasekhar $M_{c h} \sim 1.4 M_{\odot}$ determinada pela pressão dos elétrons degenerados, e a $M_{\max }$ determinada pela repulsão a altas densidades, a qual precisa atingir pelo menos o valor $M_{\max } \sim 2.1 M_{\odot}$ ou superior para dar conta das medidas listadas na Tabela 1. A linha tracejada superior é considerada um limite absoluto para as estrelas sem rotação, descritas pela Relatividade Geral e sem fontes de anisotropia ou outros efeitos, e referenciada como o limite de Rhoades-Ruffini [9]. 
Tabela 1: Algumas das maiores massas medidas em estrelas de nêutrons

\begin{tabular}{lcc}
\hline Nome & Massa $\left(M_{\odot}\right)$ & Tipo de medida \\
\hline MSP J0740+6620 & $2.14 \pm_{0.09}^{0.10}$ & Shapiro delay [11] \\
B1957+20 & $2.39 \pm_{0.29}^{0.36}$ & Modelagem da curva de luz [12] \\
Vela X-1 & $2.12 \pm 0.16$ & Modelagem da curva de luz [13] \\
PSR J2215+5135 & $2.27 \pm_{0.15}^{0.17}$ & Modelagem das linhas de emissão [14] \\
\hline
\end{tabular}

repulsão nuclear tivesse um papel importante quando a estrutura nuclear deixa de existir e a matéria é forçada a permanecer em equilíbrio a densidades acima da densidade de saturação nuclear $\rho_{0} \equiv 2.7 \times 10^{14} \mathrm{~g} \mathrm{~cm}^{-3}$ [16]. A extensão da teoria de Yukawa para estas interações repulsivas pode ser feita postulando um potencial similar com acoplamento positivo, ou seja

$$
V=+g^{2} \frac{e^{-\mu r}}{r}
$$

onde o acoplamento $\gg 1$ é medido e justifica o nome de "interações fortes". Os mésons associados à repulsão também foram observados, seus principais componentes são o $\rho(769 \mathrm{MeV})$, o $\omega(783 \mathrm{MeV})$ e o $\phi(1019 \mathrm{MeV})$. Estes mésons configuram, no limite estático, o potencial da equação 11 com um acoplamento de $\alpha_{s} \equiv \frac{g^{2}}{\hbar c} \approx 10$ (a "constante de estrutura fina"das interações fortes). O raio onde a repulsão domina totalmente, o chamado hard core, tem sido extraído recentemente [17] com o resultado $r_{0} \sim 0.35 \mathrm{fm}$.

A aplicação para a matéria acima da densidade de saturação começa pelo cálculo da energia associada às interações repulsivas. Na aproximação estática o procedimento de avaliação é bem conhecido [16] e precisa da integração do potencial no volume. Se considerarmos esquematicamente que a repulsão se estende para $r>r_{0}$, o potencial da equação 11 pode ser integrado no volume, mantendo a posição de uma partícula fixa na origem, para obter a energia de interação, com o resultado

$$
\begin{aligned}
& +g^{2} \int_{r_{0}}^{\infty} \frac{e^{-\mu\left(r-r_{0}\right)}}{\left(r-r_{0}\right)} 4 \pi r^{2} d r=+g^{2}\left[\frac{4 \pi}{\mu^{2}}\left(1-2 \mu r_{0}\right)\right. \\
& \left.+\frac{8 \pi r_{0}}{\mu^{2}} e^{2 \mu r_{0}}+4 \pi r_{0}^{2} E i\left(2 \mu r_{0}\right)\right],
\end{aligned}
$$

onde $E i$ é a integral exponencial de argumento $2 \mu r_{0} . \mathrm{Na}$ prática, este refinamento não tem efeito substancial e o resultado $\frac{4 \pi}{\mu^{2}}$ da Ref. [17] é suficientemente acurado. Uma segunda integração é necessária para obter a energia volumétrica total [16]. Com este resultado é imediato escrever a densidade de energia correspondente à repulsão $\varepsilon_{r e p}$

$$
\frac{E}{V} \equiv \varepsilon_{r e p}=\frac{2 \pi g^{2}}{\mu^{2}} n^{2} .
$$

Para efeitos práticos, utilizaremos $\mu=1 \mathrm{GeV}$ para representar uma média representativa da soma dos mésons $\rho, \omega$ e $\phi$ no potencial da equação(1).
De forma geral é imediato obter a pressão (desde que $T=0)$ ao diferenciar a equação(3)

$$
P_{\text {rep }} \equiv-\frac{\partial E}{\partial V}=\frac{2 \pi g^{2}}{\mu^{2}} n^{2}
$$

Inserindo valores numéricos e escalonando, temos que

$$
P_{\text {rep }}=2.2 \times 10^{33}\left(\frac{\alpha_{s}}{10}\right)\left(\frac{\rho}{\rho_{0}}\right)^{2} \frac{\mathrm{erg}}{\mathrm{cm}}{ }^{3}
$$

A pressão total precisa ainda da adição da componente cinética dos nêutrons livres

$$
P_{k i n}=6 \times 10^{33}\left(\frac{\rho}{\rho_{0}}\right)^{5 / 3} \frac{\mathrm{erg}}{\mathrm{cm}^{3}}
$$

Uma última corrreção decorre de introduzir via o coeficiente de $P_{k i n}$ os efeitos esperados do tratamento dos núcleons no formalismo de Hartree-Fock e a redução pelas interações de intercâmbio entre núcleons indistinguíveis. Estes efeitos reduzem pela metade a $P_{k i n}$, e assim a utilizaremos na seção seguinte. Desde que o propósito demonstrativo deste trabalho é manifesto, não é esperada uma acurácia além de um fator numérico pequeno na descrição, que é direcionada aos cursos introdutórios.

\section{Estimativa elementar do equilíbrio e as massas das estrelas de nêutrons}

Considerando a gravitação Newtoniana pelo momento, sabemos que a equação fundamental que estabelece o estado mecânico do gás é a chamada equação de equilíbrio hidrostático para uma esfera auto-gravitante

$$
\frac{d P}{d r}=-\frac{G m \rho}{r^{2}}
$$

onde $m(r)$ é a massa encerrada no raio $r$. A solução da equação (7) em termos da função pressão $P(r)$ estabelece que a estrela decorre do balanço de forças de pressão e a gravitação, que pode ser escrita genericamente como $\sum P_{i}=$ "P $P_{\text {grav }} "$, no nosso caso $\sum P_{i}=P_{\text {kin }}+P_{\text {rep }}$. Aqui a "pressão gravitacional"é uma expressão formal que resulta de variar a energia a respeito do volume que esta ocupa, de forma totalmente geral [16], ou seja, é uma quantidade com dimensões de pressão que representa a atração gravitacional oposta às pressões da matéria. 
Esta "pressão gravitacional" $P_{\text {grav }} \equiv-\frac{\partial E_{\text {grav }}}{\partial V}$ pode ser obtida de imediato escrevendo a energia gravitacional de uma esfera em função do volume $V$

$$
E_{\text {grav }}=-\frac{3}{5}\left(\frac{4 \pi}{3}\right)^{1 / 3} \frac{G M^{2}}{V^{1 / 3}}
$$

com o resultado

$$
P_{\text {grav }} \equiv-\frac{\partial E_{\text {grav }}}{\partial V}=C M^{2 / 3} \rho^{4 / 3}
$$

com $C=\frac{G}{5}\left(\frac{4 \pi}{3}\right)^{1 / 3}$ e um fator adicional multiplicativo 1.2 que simula o efeito da gravitação mais intensa da Relatividade Geral. Escalonando para valores de referência temos finalmente

$$
P_{\text {grav }}=3.2 \times 10^{33}\left(\frac{M}{M_{\odot}}\right)^{2 / 3}\left(\frac{\rho}{\rho_{0}}\right)^{4 / 3} \frac{\mathrm{erg}}{\mathrm{cm}^{3}} .
$$

Estamos agora em condições de estimar as condições físicas para a existência de soluções de equilíbrio utilizando a expressão (5) já obtida para a repulsão, adicionando o termo de energia cinética do gás de nêutrons livres equação(6) com $\rho=m_{n} n$. Note-se que este termo $P_{k i n}$ é o único considerado por Landau na sua estimativa inicial das estrelas de nêutrons [5]. Porém, na soma das pressões $P_{\text {grav }}=P_{k i n}+P_{\text {rep }}$ o segundo termo (repulsivo) é quadrático e domina além do ponto onde $P_{k i n} \cong P_{r e p}$. Escrevendo explicitamente os termos e igualando

$3 \times 10^{33}\left(\frac{\rho}{\rho_{0}}\right)^{5 / 3} \frac{\mathrm{erg}}{\mathrm{cm}^{3}}=2.3 \times 10^{33}\left(\frac{\alpha_{s}}{10}\right)\left(\frac{\rho}{\rho_{0}}\right)^{2} \frac{\mathrm{erg}}{\mathrm{cm}^{3}}$,

vemos que a dominância de $P_{\text {rep }}$ acontece acima da densidade $\rho=2.5 \rho_{0}$. Portanto, o equilíbrio requer $P_{\text {grav }} \approx$ Prep

$5 \times 10^{33}\left(\frac{\rho}{\rho_{0}}\right)^{4 / 3} \frac{\mathrm{erg}}{\mathrm{cm}^{3}}=2.3 \times 10^{33}\left(\frac{\alpha_{s}}{10}\right)\left(\frac{\rho}{\rho_{0}}\right)^{2} \frac{\mathrm{erg}}{\mathrm{cm}^{3}}$,

com solução em $\rho=4.5 \rho_{0}$ e $M \approx 2 M_{\odot}$ (vide a seguir). Esta densidade deve corresponder às regiões centrais de uma estrela de nêutrons de pelo menos $\sim 2.1 M_{\odot}$ para dar conta das observações. A situação se ilustra na Figura 2.

Com esta valor da densidade, a qual deve ser propriamente interpretada como uma média espacial na região central, podemos estimar de imediato a massa máxima dentro de um erro percentual pequeno na aproximação de densidade constante, a qual resulta em

$$
M_{\text {max }}=2.54\left(\frac{\rho}{4.5 \rho_{0}}\right)\left(\frac{R}{10 k m}\right)^{3} M_{\odot} .
$$

A equação 13 não deve ser adotada literalmente, já que as aproximações se combinaram para dar um resultado razoável. Para uma predição mais refinada, é necessário integrar as equações de estrutura de Tolman-OppenheimerVolkoff deduzidas para soluções estáticas com simetria

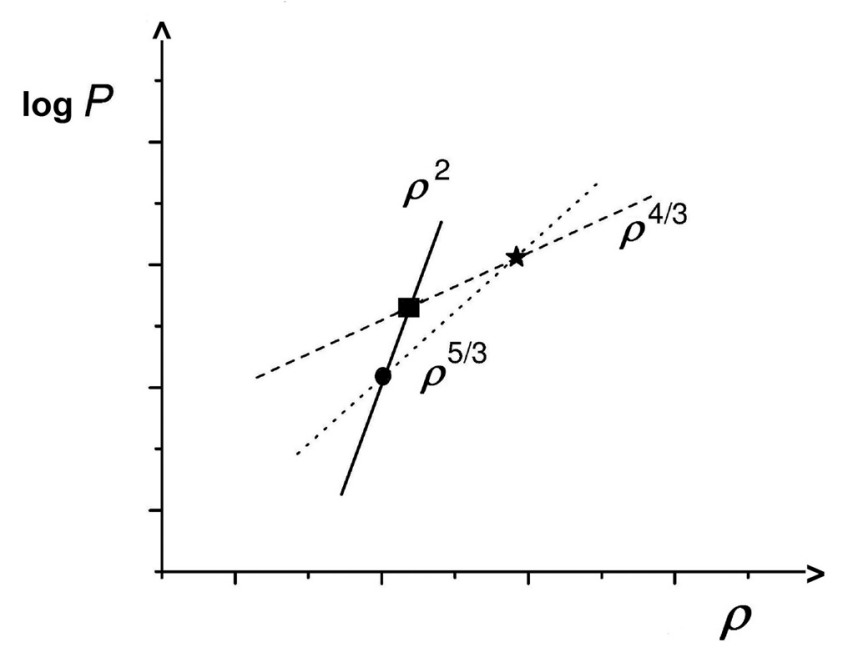

Figura 2: Esquema básico das pressões e o equilíbrio para a matéria dentro das estrelas de nêutrons. A expressão $P_{k i n} \propto \rho^{5 / 3}$ da energiia cinética dos nêutrons livres (a utilizada pelo Landau [5] na sua estimativa inicial, tracejada curta) é dominada pela pressão das interações repulsivas $P_{r e p} \propto \rho^{2}$ (atribuidas totalmente ao méson $\omega$ por simplicidade, linha cheia) no ponto indicado com o círculo preto. Assim, o equilíbrio da estrela acontece quando esta pressão $P_{r e p}$ balança a "pressão gravitacional"(vide texto, linha tracejada longa) no ponto indicado pelo quadrado preto. As massas obtidas são suficientes para explicar os valores da Tabela 1, embora este modelo é muito simplificado. É importante destacar que a expressão das interações repulsivas não pode ficar acausal: a condição $\left(\frac{\partial P}{\partial \rho}\right)^{1 / 2} \leq c$ mostra que isto acontece para $\rho \gg 10 \rho_{0}$ e a utilização da expressão (5) não gera problemas.

esférica na Relatividade Geral. Soluções apropriadas para o caso de uma equação de estado quadrática podem ser consultadas nas Refs. [18] e [19]. Mas ainda sem entrar a fundo em este problema, a descoberta de Lattimer e Prakash [20] a respeito da existência de um limite superior à densidade central de qualquer modelo estelar pode ser utilizada. No trabalho da Ref. [20] é mostrado que todas as soluções exatas conhecidas tem como envelope a solução denominada Tolman VII, a qual começa com o ansatz funcional para a densidade $\rho=\left(1-\left(\frac{r}{R}\right)^{2}\right)$, aplicável à estrelas de nêutrons "normais"(com densidade zero na superfície), e que depois da integração das equações de Tolman-Oppenheimer-Volkoff expressa a densidade central como $\rho_{c}=1.45 \times 10^{16}\left(\frac{M_{\odot}}{M}\right)^{2}$. Esta curva determina um limite superior para $\rho_{c}$ quando inserida a maior massa medida na Natureza. No caso de adotarmos a massa de B1957+20 da Tabela 1 , este valor é de $\approx 9 \rho_{0}$, dentro de um fator 2 do que obtivemos acima de forma elementar. Concluimos que para propósitos didáticos é suficiente o cálculo apresentado que ilustra bem os princípios físicos e os fatores importantes da determinação da estrutura estelar relativísitica no regime extrema das estrelas de nêutrons. 


\section{Conclusões}

O objetivo deste trabalho tem sido esclarecer que no regime de alta densidade, o das estrelas de nêutrons, a massa de Chandrasekhar derivada para as anãs brancas não tem efetivamente nenhum papel, contrariamente às afirmações em várias referências específicas. Ainda mais, a existência de estrelas de nêutrons com massas medida acima de $2 M_{\odot}$ refuta esta ideia desde o ponto de vista empírico. O fato de que existe uma massa máxima para as estrelas de nêutrons decorre da presença da pressão como fonte da gravitação na Relatividade Geral. Assim, a instabilidade na estrutura é causada pela forma da equação de equilíbrio de Tolman-Oppenheimer-Volkoff, e a massa máxima é escrita às vezes como "massa TOV", $M_{T O V} \equiv M_{\max }$ na literatura.

Temos adotado um cenário muito simples para tratar a repulsão nuclear, a qual é a verdadeira responsável pela estabilidade estelar nesse regime, e mostrado que explica a presença de estrelas de nêutrons de grande massa. É claro que este tratamento não pode ser considerado como muito mais que uma parametrização, mas contém os elementos básicos necessários para entender como as forças repulsivas atuam na prática. Acreditamos que este tipo de abordagem pode ser útil para um curso introdutório de Astrofísica Nuclear ou mesmo de Astronomia Geral onde a simbiose entre a Física e Astronomia tenha um tratamento e destaque merecido.

\section{Agradecimentos}

O autor agradece ao CNPq (Brasil) pela concessão de uma Bolsa PQ e a FAPESP (São Paulo) pelo suporte através do Projeto Temático 13/26258-4.

\section{Referências}

[1] R.H. Fowler, Mont. Not. R.A.S 87, 114 (1926).

[2] J.E. Horvath, Fundamentos de Evolução Estelar, Supernovas e Estrelas Compactas (Livraria da Física, São Paulo, 2011).

[3] S. Chandrasekhar, An Introduction to the Study of Stellar Evolution (Dover Publications, Nova York, 1939)

[4] C. Raithel, T. Sukhbold e F. Özel, Astrophys. J. 856, 35 (2018).

[5] L.D. Landau, Phys. Z. Sowjetunion 1, 152 (1932).

[6] D.G. Yakovlev, P. Haensel, G. Baym e C. Pethick, Phys. Usp. 56, 289 (2013).

[7] A. Hewish, S.J. Bell, J.D.H. Pilkington, P.F. Scott e R.A. Collins, Nature 217, 709 (1968).

[8] J.E. Horvath e R. Valentim, Handbook of Supernovae (Springer, New York, 2017).

[9] C.E. Rhoades e R. Ruffini, Phys. Rev. Lett. 32, 324 (1974).

[10] R. Valentim, E. Rangel e J.E. Horvath, Mont. Not. R.A.S 414, 1427 (2011).

[11] H.T. Cromartie, E. Fonseca, S.M. Ransom, P.B. Demorest, Z. Arzoumanian, H. Blumer, P.R. Brook, M.E.
DeCesar, T. Dolch, J.A. Ellis et al. Nature Astronomy 4, 72 (2019).

[12] M.H. van Kerkwijk, R. Breton e S.R. Kulkarni, Astrophys. J. 728, 95 (2011).

[13] M. Falanga, E. Bozzo, A. Lutovinov, J.M. BonnetBidaud, Y. Fetisova e J. Puls, Astron. \& Astrophys. 577, A130 (2015).

[14] M. Linares, T. Shahbaz e J. Casares, Astrophys. J. 859, $54(2018)$

[15] H. Yukawa, Proc. Phys.-Math. Soc. Jpn. 17, 48 (1935).

[16] S. Shapiro e S.L. Teukolsky, Black Holes, White Dwarfs, Neutron Stars: The Physics of Compact Objects (Wiley, New York, 1983).

[17] K.A. Bugaev, A.I. Ivanytskyi, V.V. Sagun, B.E. Grinyuk, D.O. Savchenk, G.M. Zinovjev, E.G. Nikonov, L.V. Bravin, E.E. Zabrodin, D.B. Blaschke et al., Universe 5, 63 (2019).

[18] T.Feroze e A. Siddiqui, Gen. Rel. Grav. 43, 1025 (2011).

[19] M. Malaver, Frontiers of Mathematics and Its Applications 1, 9 (2014).

[20] J.M. Lattimer e M. Prakash, Phys. Rev. Lett. 94, 111101 (2005). 